\title{
Pathophysiology, diagnosis and management of nephrogenic diabetes insipidus
}

\section{Detlef Bockenhauer and Daniel G. Bichet}

University College London Institute of Child Health, 30 Guilford Street, London, WC1N 1EH, UK (D.B.). Departments of Medicine and Molecular and Integrative Physiology, Université de Montréal, Research Center, Hôpital du Sacré-Coeur de Montréal, 5400 Boulevard Gouin Ouest, Montréal, H4J 1C5 Canada (D.G.B.).

Correspondence to:

D.G.B.

daniel.bichet@umontreal.ca

Key points

- Nephrogenic diabetes insipidus (NDI) is caused by inability of the kidneys to concentrate urine by reabsorption of water in the collecting duct

- NDI can be inherited (X-linked or autosomal) or acquired, most commonly as a result of lithium treatment

- Management of primary forms of NDI focuses on dietary modification to reduce osmotic load and pharmacological treatment with prostaglandin synthesis inhibitors and thiazide diuretics

- With appropriate treatment, complications of NDI, such as failure to thrive and mental retardation resulting from repeated hypernatraemic dehydration, can be avoided

- New treatment approaches for congenital NDI have been tested in animal models, but efficacy in patients has not yet been confirmed 


\section{Abstract}

Healthy kidneys maintain fluid and electrolyte homoeostasis by adjusting urine volume and composition according to physiological needs. The final urine composition is determined in the last tubular segment: the collecting duct. Water permeability in the collecting duct is regulated by arginine vasopressin (AVP). Secretion of AVP from the neurohypophysis is regulated by a complex signalling network that involves osmosensors, barosensors and volume sensors. AVP facilitates aquaporin (AQP)-mediated water reabsorption via activation of the vasopressin V2 receptor (AVPR2) in the collecting duct, thus enabling concentration of urine. In nephrogenic diabetes insipidus (NDI), inability of the kidneys to respond to AVP results in functional AQP deficiency. Consequently, affected patients have constant diuresis, resulting in large volumes of dilute urine. Primary forms of NDI result from mutations in the genes that encode the key proteins AVPR2 and AQP2, whereas secondary forms are associated with biochemical abnormalities, obstructive uropathy or the use of certain medications, particularly lithium. Treatment of the disease is informed by identification of the underlying cause. Here we review the clinical and diagnostic aspects of NDI, the various aetiologies, current treatment options and potential future developments.

\section{[H1] Introduction}

Nephrogenic diabetes insipidus (NDI) results from failure of the kidneys to concentrate urine. Patients with this disease typically produce around $12 \mathrm{l}$ of urine per day. Assuming a bladder volume of $500 \mathrm{ml}$, this volume of urine will necessitate voiding approximately once per hour throughout the day and night. 
Moreover urinary fluid losses need to be replenished by constant fluid intake. The disease has a substantial impact on quality of life as sleep is frequently interrupted and patients must plan their daily activities with logistics for frequent voiding and access to drinking water.

Primary and secondary forms of NDI exist. Paediatricians typically see patients with primary inherited forms of the disease, mostly boys presenting in the first year of life with failure-to-thrive and vomiting. By contrast, in adults acquired NDI is much more common than primary NDI. Medical treatment can ameliorate the symptoms of NDI and as understanding of the underlying pathophysiology improves, new potential treatment options arise. In this Review we consider the clinical, diagnostic and therapeutic aspects of NDI in the context of the physiology of renal water handling to enable a molecular understanding of the disease. We also discuss recent insights into the regulation of water permeability and their consequences with respect to diagnosis and new treatment options.

\section{[H1] Physiology of urine concentration}

Healthy adult kidneys produce approximately $180 \mathrm{l}$ of primary glomerular filtrate per day. ${ }^{1}$ The vast majority of this filtrate is reabsorbed in the proximal tubule, which is freely permeable to water owing to the constitutive expression of aquaporin (AQP)-1 water channels. ${ }^{2}$ As solutes are reabsorbed in the proximal tubule, water follows passively along the osmotic gradient. The remaining urine is thus still isotonic when it enters the loop of Henle, the key segment for counter-current concentration (Figure 1). Urine concentration begins in the thin descending limb (TDL). Mechanisms of concentration include AQP1-mediated 
exit of water into the medullary interstitium. ${ }^{3}$ However, data from several rodent models show that Aqp1 expression is mainly restricted to the first $60 \%$ of the TDL rather than to the deeper papillary parts in which the steepest part of the osmotic gradient is generated. ${ }^{4}$ Concentration of the tubular fluid in these innermost parts of the TDL might occur via passive sodium influx, ${ }^{5}$ but no substantial sodium permeability has been observed. ${ }^{6}$ Thus, the exact mechanism of urinary concentration in the innermost part of the medulla remains to be elucidated.

Urine subsequently enters the thick ascending limb (TAL, also known as the diluting segment), which is impermeable to water but actively removes sodium chloride via the co-transporter solute carrier family 12 member 1 (SLC12A1, also known as NKCC2), thereby diluting the urine. ${ }^{7}$ The accumulation of solutes in the interstitium generates the driving force for the removal of water from the TDL (in long-looped nephrons) and the entry of sodium chloride (in short-looped nephrons), completing the counter-current multiplier.

Further removal of sodium chloride occurs in the distal convoluted tubule via SLC12A3 (also known as NCC). At entry into the arginine vasopressin (AVP)sensitive connecting tubules and collecting ducts, urine osmolality is typically around 50-100 mOsm $/ \mathrm{kg}$. The final osmolality of the urine is solely dependent on the availability of water channels. If these channels are present, water exits the tubule following the interstitial concentration gradient and the urine is concentrated. If no water channels are present, dilute urine will be excreted. 


\section{[H1] Vasopressin-regulated water permeability}

Vasopressin secreted by hypothalamic neurons in response to changes in soma volume (Figure 2) is the key regulator of the water permeability of the collecting ducts. The key cell type that mediates this regulated water permeability is the principal cell, which constitutively expresses AQP3 and AQP4 on the basolateral side. $^{2}$ The water permeability of the apical membranes of the principal cell is determined by the availability of AQP2 water channels. In the absence of AVP signalling, these channels are located in intracellular vesicles and no apical water permeability exists. ${ }^{8}$ Binding of AVP to the vasopressin V2 receptor (AVPR2) initiates a signalling cascade that ultimately leads to the insertion of AQP2 channels into the apical membrane(Figure 3). AVPR2 is a G-protein-coupled receptor and binding of AVP leads to the release of stimulatory G-protein, which in turn activates the neighbouring adenylyl cyclase. ${ }^{9}$ The resulting increase in levels of cAMP activates protein kinase A (PKA).

The cytoplasmic carboxy-terminal tail of AQP2 has five canonical PKAphosphorylation sites: Thr244, Ser256, Ser261, Ser264 and Thr269 (Ser269 in mice). ${ }^{10}$ Upon administration of the vasopressin analogue 1-desamino-8-Darginine vasopressin (DDAVP), phosphorylation of AQP2 at Ser256, Ser264 and Ser269 is abundantly increased, whereas phosphorylation at Ser261 is decreased; ${ }^{10}$ specific phosphorylation of these residues might serve distinct physiological roles. In a mammalian cell line (LLC-PK1) transiently transfected with AQP2, phosphorylation at Ser256 was essential for trafficking of the water channel to the plasma membrane, ${ }^{11}$ whereas in Xenopus oocytes, phosphorylation of at least three subunits of the AQP2 tetramer at Ser256 was 
required for redistribution from storage vesicles to the apical plasma membrane. ${ }^{12}$ The critical role of Ser256 in AQP2 function was confirmed by the identification of mutations that impair phosphorylation at this site in patients with NDI.13,14 In vitro studies demonstrated the failure of such mutants to reach the apical membrane. ${ }^{13,14}$

AVP also induces phosphorylation of AQP2 at Ser269, but this action is delayed and requires prior phosphorylation at Ser265.15 Phosphorylation at Ser269 might increase retention of AQP2 at the apical plasma membrane and interactions of AQP2 with proteins participating in the endocytic machinery. ${ }^{16} \mathrm{By}$ contrast, AQP2 phosphorylated at Ser261 is found mainly in intracellular vesicles; phosphorylation at this site is thought to stabilize ubiquitination of AQP2 ${ }^{17}$ at Lys270. ${ }^{18}$ Ubiquitination might, therefore, mediate the endocytosis of AQP2 from the plasma membrane in the absence of AVP stimulation. This mechanism leads to a limited tenure of AQP2 in the apical membrane of principal cells; endocytosis of this water channel enables the cells to return to their waterimpermeable state. The role of AQP2 phosphorylation at Thr244 is unknown.

The actions of cAMP might not only be mediated via the classical PKA/cAMPdependent protein kinase pathway, but also by guanine nucleotide-exchange factors such as exchange protein directly activated by cAMP (Epac). ${ }^{19}$ Like PKA, Epac contains an evolutionally conserved cAMP-binding domain that senses intracellular cAMP levels and acts as a molecular switch to control diverse biological functions. ${ }^{20}$ Epac might be involved in the long-term regulation of AQP2 abundance, whereas PKA has independent short-term effects. ${ }^{21}$ 
In addition to an acute role in AQP2 localization, AVP-mediated increases in cAMP promote AQP2 expression via PKA-mediated phosphorylation of the cAMP responsive element binding protein, which subsequently stimulates transcription from the AQP2 promoter. ${ }^{22-24}$ The change in localization of AQP2 occurs within minutes, whereas the increase in transcription requires hours to take effect. ${ }^{25,26}$

\section{[H1] Congenital NDI}

In 1992 the AVPR2 gene that encodes the AVP2 receptor was cloned and mutations in this gene were identified in patients with X-linked NDI (Figure 4). ${ }^{27-}$ 30 The $A Q P 2$ gene was cloned in $1993^{31,32}$ and in 1994 mutations in $A Q P 2$ were found to underlie autosomal recessive NDI. ${ }^{33}$ The discovery of these two key genes-AVPR2 and $A Q P 2$ - has enabled genetic testing of affected patients. Mutations in either of these genes are identified in almost all patients with a clear clinical phenotype of congenital NDI. ${ }^{34,35}$

\section{[H2] AVPR2 mutations}

Approximately $90 \%$ of patients with inherited NDI have a mutation in AVPR2.36,

37 As this gene is located on the X-chromosome, AVPR2 mutations have an Xlinked pattern of inheritance; consequently the majority of patients with NDI are male. Occasionally, female patients with NDI due to AVPR2 mutations have been described. ${ }^{34,35,38-41}$ In one study, 16 of 64 (25\%) female carriers of AVPR2 mutations showed polyuric symptoms and four of these patients $(6 \%)$ were diagnosed with complete NDI rather than the milder partial NDI phenotype described below. ${ }^{34}$ Skewed X-inactivation is thought to cause symptoms in some female carriers of $A V P R 2$ mutations, although these symptoms do not necessarily 
correlate with the X-inactivation patterns observed in leukocytes. ${ }^{42}$ Presumably, X-inactivation patterns can differ between tissues, so the pattern in leukocytes might not reflect that in the kidney. In our experience at Great Ormond Street Hospital, London, UK, two female relatives in 20 families with AVPR2 mutations have been diagnosed with complete NDI (unpublished data). One of these women was the index case in her family, but not all female carriers have been systematically investigated. Partial NDI symptoms were observed in two other female carriers in one family. 43

A few AVPR2 mutations have been identified recurrently owing to a founder effect. For example, the Hopewell mutation (Trp71X)—derived from an Ulster Scot immigrant who arrived in Halifax, Canada on the ship Hopewell in 1761has been identified in $>40$ North American patients. ${ }^{44,45}$ Another recurrent mutation is the Cannon mutation (Leu312X), which is named after a large pedigree with $\geq 38$ affected patients in Utah. ${ }^{45}$ Most patients, however, have individual mutations; to date $>250$ different $A V P R 2$ mutation have been described in >300 families ( ${ }^{46}$ and our unpublished data). Interestingly, most of these mutations are missense and, when assessed in vitro, seem to encode functional but misfolded receptors, which are retained and degraded in the endoplasmic reticulum (ER). ${ }^{47}$

\section{[H3] AQP2 mutations}

Approximately $10 \%$ of cases of congenital NDI are due to loss-of-function mutations in the $A Q P 2$ gene located on chromosome $12.45,34,48$ These mutations are typically inherited in an autosomal recessive fashion, although a few mutations have been described to cause autosomal dominant disease. ${ }^{13,49-51}$ 
AQP2 is a homotetramer; four subunits assemble to form the water channel. Dominant mutations typically affect amino acids in the C-terminus, leading to aberrant trafficking; mutant AQP2 subunits are able to oligomerize with wildtype protein to form tetramers, but exert a dominant-negative effect on trafficking of these assembled tetramers. ${ }^{52}$ Interestingly, specific mutations direct AQP2 trafficking to distinct cellular compartments, such as the Golgi complex, ${ }^{13}$ late endosomes and lysosomes ${ }^{49}$ or the basolateral membrane. ${ }^{51,53}$ These findings have provided insights into the intracellular trafficking motifs of AQP2.

\section{[H2] Epidemiology}

Few population-based data on the incidence of congenital NDI exist, but estimates can be made. In 2000 we assumed that we had complete ascertainment of all the patients with AVPR2 mutations who were born in the province of Quebec, Canada between 1988 and 1997.35 Indeed, in the subsequent 18 years, no patients with $A V P R 2$ mutations born in the province during this period have emerged. Based on these data, we estimated the incidence of Xlinked NDI in the general population of Quebec to be four in 454,629 or approximately 8.8 per million (SD 4.4 per million) male live births. This estimate might be representative of the general world population. However, owing to chance population genetic events, such as a founder effect, the incidence of NDI is elevated in certain regions. For example, in the Canadian maritime provinces of Nova Scotia and New Brunswick, we estimate the incidence of the Hopewell mutation to be 58 per million male live births. ${ }^{35} \mathrm{~A}$ higher incidence of NDI is also found in Utah due to the prevalence of the Cannon mutation. ${ }^{44}$ 
Based on the families referred to us for genetic testing, we estimate that the incidence of NDI secondary to $A Q P 2$ mutations is five to ten times lower than that of NDI secondary to AVPR2 mutations. The incidence of this type of congenital NDI might, however, be increased in populations with a high degree of consanguinity; we have found an over-representation of the Val71Met $A Q P 2$ allele in patients of Pakistani descent. ${ }^{54}$

\section{[H1] Secondary inherited forms of NDI}

In addition to primary forms of congenital NDI a few cases of secondary inherited NDI have been reported. 55,56 These patients have Mendelian diseases that affect tubular function with NDI as a secondary complication. In some such patients the NDI symptoms dominate the clinical picture, leading to an initial misdiagnosis, with the true underlying cause of disease subsequently identified. ${ }^{55,57}$ Most of the primary diseases associated with secondary NDI, such as Bartter syndrome and apparent mineralocorticoid excess, are associated with hypokalaemia and hypercalciuria. These associations might provide clues to the aetiology of NDI, as both hypokalaemia and hypercalciuria are associated with decreased AQP2 expression. ${ }^{58,59}$ Hypercalciuria has been suggested to cause a urinary concentration defect mediated by the calcium-sensing receptor, which is expressed on the luminal side of collecting duct cells and is thought to affect AQP2 trafficking by altering cAMP levels. ${ }^{60,61}$

\section{[H1] Acquired NDI}

In adult nephrology practice the majority of patients who present with NDI have acquired this disease, with lithium treatment being the predominant cause. ${ }^{62-64}$ Reports of NDI among patients treated with lithium vary widely but the incidence of this adverse effect has been reported to be as high as $85 \%{ }^{63,65}$ As 
$0.25-0.77 \%$ of the general population aged $>65$ years are prescribed lithium, it is not surprising that lithium-induced NDI is fairly commonly. ${ }^{66,67}$ Indications for lithium treatment include bipolar disorder, schizoaffective disorder, depression, alcoholism and cluster headaches. ${ }^{64}$ Cessation of lithium therapy can resolve the symptoms of NDI, but this approach is not an option in most cases because the beneficial effects of the drug on the psychiatric disorder outweighs the negative impact of the polyuric complications on quality of life.

In rats, chronic lithium administration ( $>4$ weeks) led to epithelial remodelling in the collecting duct and a dramatic reduction in the number of principal cells. ${ }^{6}$ Moreover, in the mouse collecting duct cell line mpkCCD, lithium exposure decreased Aqp2 abundance independent of cAMP levels. ${ }^{69}$ Consistent with these data, decreased urinary excretion of AQP2 has been observed in patients with lithium-induced NDI. ${ }^{70}$ The exact mechanism of lithium toxicity on the principal cell remains to be elucidated, but robust data suggest that lithium exerts its effect after entering the cell through epithelial sodium channels (ENaCs), which have a high permeability for lithium. ${ }^{10}$ Consequently, treatment with ENaC blockers, such as amiloride, can increase urine osmolality and ameliorate polyuria in lithium-induced NDI. ${ }^{71-73}$

Other acquired causes of typically transient NDI include hypercalcaemia, hypercalciuria and obstructive uropathy. In patients with obstructive uropathy, NDI is thought to result from direct suppression of AQP2 expression, which might be mediated by hydrostatic pressure. Animal studies have shown decreased Aqp2 expression in the setting of bilateral ureteric obstruction, ${ }^{74}$ 
whereas in unilateral obstruction a marked decrease in Aqp2 is seen only in the obstructed kidney. ${ }^{75}$ This down-regulation of Aqp2 persists up to 30 days after release of obstruction, perhaps explaining the diuresis often observed after release of severe obstruction in patients. ${ }^{9}$

\section{[H1] Initial presentation}

Most patients with congenital NDI present with failure to thrive during the first few months of life, whereas those with acquired NDI typically present later in life with polyuria and or polydipsia. In patients with congenital NDI the antenatal history is typically normal with no polyhydramnios, which helps to distinguish NDI from other polyuric disorders, such as Bartter syndrome. Parents often report that the baby sucks vigorously but vomits shortly afterwards. This vomiting is thought to occur because intake of large fluid volumes causes gastrooesophageal reflux.

NDI is a rare disease so does not feature prominently on the diagnostic radar of frontline medical staff. Families seeking medical attention for their yet undiagnosed baby might, therefore, be sent home without further investigations because the attending professionals are falsely reassured by the large urine output. Laboratory investigations in such instances would show the typical picture of hypernatraemia with inappropriately dilute urine, the biochemical "fingerprint" of diabetes insipidus. If blood tests are obtained and hypernatraemia is noted without consideration of a urinary concentration defect, inappropriate treatment with $0.9 \%$ saline might be instigated because of concerns regarding too rapid correction of the hypernatraemia. Unfortunately, 
given the imbalance between the sodium concentration of the administered fluid (154 mmol/l) and the urine (usually $<10 \mathrm{mmol} / \mathrm{l}$ ), this approach will result in even higher plasma sodium concentrations.

\section{[H1] Diagnosis}

In patients who present with hypernatraemic dehydration and dilute urine, a DDAVP test can help to distinguish between cranial diabetes insipidus (which is normally the result of disease of the hypothalamus or surrounding tissues) and NDI. In patients with suspected diabetes insipidus who present with polyuria and normal plasma biochemistries, a water deprivation test might first be performed to challenge urinary concentration.

[H2] DDAVP test

As DDAVP has high specificity for AVPR2 it can be used to assess the renal response whilst avoiding AVPR1-mediated vasoconstriction. DDAVP is available in formulations suitable for oral, intranasal, subcutaneous or intravenous (IV) administration. After DDAVP administration a urine osmolality $>800 \mathrm{~m} 0 \mathrm{sm} / \mathrm{kg}$ is usually considered normal and excludes a diagnosis of NDI, whereas a urine osmolality below plasma osmolality indicates AQP2 deficiency and is consistent with the diagnosis. Care needs to be taken in the interpretation of intermediate results, especially in infants, as full urinary concentrating ability develops during the first year of life. ${ }^{76}$ Other causes for submaximal values include interstitial renal disease, use of loop diuretics, partial NDI, washout of the interstitial medullary concentration gradient or technical failure of the test, for example as a result of incomplete DDAVP absorption. 
Various protocols for the DDAVP test exist, but we prefer to use IV administration to enable the most reliable absorption of the polypeptide. ${ }^{77}$ The DDAVP dose $(0.3 \mathrm{mcg} / \mathrm{kg})$ used in this test is the same as that used to boost factor VIII concentration in patients with mild haemophilia or von Willebrand disease. ${ }^{78,79}$ The key risk of the test is the development of hyponatraemia in patients who respond to DDAVP and keep drinking. The physiological suppression of thirst from reduced plasma osmolality prevents this complication in the vast majority of responders, but patients with habitual polydipsia and babies who keep receiving feeds from their carer are at risk. Strict observation of fluid balance is required to prevent this potentially serious complication and patients should only be allowed to receive a fluid volume equivalent to their ongoing losses.

A key advantage of IV administration is the short half-life of DDAVP in blood, which necessitates an observation time of only $2 \mathrm{~h}$, thus minimizing the burden of the test. Other advantages include a low risk of test failure owing to incomplete absorption and the ability to distinguish between X-linked and autosomal-recessive forms of NDI, as AVPR2 mediates haemodynamic and coagulation factor changes in response to high-dose DDAVP.77 This response is abrogated in patients with defective AVPR2 function, yet retained in those with mutations in $A Q P 2$. The low plasma levels of DDAVP achieved with other forms of administration make detection of these subtle changes virtually impossible.

\section{[H1] Spectrum of clinical disease}

\section{[H2] Mental impairment}


As with most inherited diseases a wide spectrum of severity of clinical disease is observed in NDI. The most severe consequences are seen in patients who have not received adequate treatment and have experienced repeated episodes of hypernatraemic dehydration, leading to brain damage and impaired mental development. $^{39,80,81}$ In some such patients, intracranial calcifications can be observed. These calcifications are thought to result from endothelial cell necrosis during severe dehydration and thus might represent a radiological correlate to brain damage from recurrent hyperosmolar stress associated with hypernatraemic dehydration. 82

Fortunately, cases of severe mental impairment in patients with NDI are now virtually non-existent in countries where modern health care is available. Nevertheless, impaired school performance and behavioural abnormalities are often reported among patients with NDI. In a Dutch study, 8 of 17 participants were diagnosed with attention deficit hyperactivity disorder. ${ }^{83}$ This diagnosis might be partly related to the constant craving for water and need to void. Some data, however, suggest that behavioural abnormalities might be an intrinsic aspect of the disease. Vasopressin (and oxytocin) act as neurotransmitters that modulate the autonomic fear response $\mathrm{e}^{84}$ and vasopressin has been described to enhance aggressiveness, anxiety and stress levels in an animal model ${ }^{85}$ Raised vasopressin levels might also potentially affect long-term behaviour in patients with NDI.

\section{[H2] Flow uropathy}

Dilatation of the urinary tract is a recurrently noted complication of NDI and patients with poor voiding habits are especially at risk. ${ }^{39,86,87}$ In those with 
hydronephrosis, anatomic causes of obstruction should also be considered. Such obstructions are potentially remediable and even minor impediments to urine flow can cause severe dilatation in this polyuric disorder. ${ }^{88}$ Obstructive nephropathy can be seen in cranial diabetes insipidus and NDI, and if untreated can lead to obstructive end-stage renal disease.

\section{[H2] Partial NDI}

Some patients with congenital NDI have a mild phenotype; they present after infancy with normal development, often for the assessment of polyuria or enuresis, and typically show intermediate urine osmolality after DDAVP administration (greater than plasma osmolality but $<800$ mosm $/ \mathrm{kg}$ ). Patients with partial NDI typically carry mutations that result in partial function of either AVPR2 ${ }^{43,89}$ or AQP2. ${ }^{90,91}$ The first mutation to be associated with partial NDI was the Asp85Asn mutation in AVPR2.89 Interestingly, another missense mutation, Val88Met, has been associated with partial NDI in some patients and complete NDI in others. ${ }^{43,}$ 92, 93 The underlying disease mechanism in patients with these missense mutations is ER retention of the mutant protein, but degree to which the mutant protein can escape from this retention and traffic to the cell membrane seems to differ between individual patients.

\section{[H1] Treatment}

Current treatment approaches for congenital NDI focus on amelioration of the symptoms rather than curing the disease. If possible, treatment of acquired NDI should target the underlying cause, such as relief of urinary obstruction or amiloride therapy in lithium-associated NDI. ${ }^{73}$ If such approaches are not possible, treatment of congenital NDI is comparable to that of primary NDI.

\section{[H2] Management of hypernatraemic dehydration}

Most emergency protocols for any patient with hypernatraemic dehydration 
suggest initial treatment with $0.9 \%$ saline, owing to concerns about lowering plasma sodium levels too rapidly. The situation differs, however, in patients with NDI because of the ongoing losses of essentially pure water with the urine; infusion of $0.9 \%$ saline will result in excess sodium chloride administration and thus worsen the hypernatraemia. In these patients, isotonic fluids should be reserved only for acute intravascular volume expansion in hypovolaemic shock, which is an exceedingly rare complication, as extracellular fluid volume is usually sufficiently preserved in hypernatraemia. Thus, patients with NDI should be treated with hypotonic fluids, either enterally (using water or milk) or if necessary intravenously (using 5\% dextrose in water). Hypotonic fluids must never be administered as an intravenous bolus; instead, the infusion rate should only slightly exceed the urine output. The aim is to provide just enough water to safely normalize plasma sodium concentration at a rate of $<0.5 \mathrm{mmol} / \mathrm{l}$ per $\mathrm{h}$ $(<10-12 \mathrm{mmol} / \mathrm{l}$ per day $)$. The main risk of a rapid decrease in plasma sodium is cerebral oedema and potentially death. ${ }^{94-97}$ Consequently, careful fluid balance and frequent monitoring of clinical state and biochemistries is key to safe treatment and requires a clinical environment with sufficient experience in the treatment of complicated electrolyte disorders. Fluids should be taken orally as soon as feasible, to enable the thirst physiology to properly regulate fluid intake. As dextrose in water provides no osmotic load (Box 1), urine output can decrease substantially, highlighting the importance of ongoing monitoring and fluid balance to avoid rapid swings in plasma sodium concentrations.

\section{[H2] Diet}

Dieticians have a key role in the management of patients with NDI, especially in the first year of life when intake of fluid and calories is coupled. Minimizing the 
osmotic load (Box 1), whilst providing the recommended caloric and protein intake to enable normal growth and development is a cornerstone of paediatric NDI management.

\section{[H2] Thiazide diuretics}

The use of diuretics in polyuric disorders seems counterintuitive. The therapeutic potential of these agents in NDI was discovered more or less serendipitously, but reported in patients as early as $1905 .{ }^{98}$ This observation was confirmed in the 1950s when administration of hydrochlorothiazide was shown to reduce urine output in experimental animals with diabetes insipidus by as much as $50 \% .{ }^{99}$ An early study in patients with NDI showed a small reduction in urine volume with concomitant increases in urine osmolality and sodium shortly after commencing hydrochlorothiazide treatment. ${ }^{100}$ After 3 days of treatment, sodium excretion had decreased to less than baseline, whereas urine output had decreased to $\sim 50 \%$ of baseline. These changes were associated with reductions in plasma volume and body weight. The researchers astutely concluded that the initial increase in urine osmolality was related to inhibition of urinary dilution, mediated by decreased salt reabsorption through the thiazidesensitive co-transporter SLC12A3 in the distal tubule, and that the loss of sodium led to a reduction in plasma volume with subsequent enhanced proximal reabsorption of the glomerular filtrate, so that less water was presented to the collecting duct and lost in the urine.

A SLC12A3-independent mechanism of thiazide-mediated reductions in urine output was proposed based on studies in Slc12a3 knock-out mice with lithiuminduced NDI.101 A marked reduction in urine volume with unchanged urine 
osmolality was observed in these mice after hydrochlorothiazide administration. The researchers speculated that this reduction might be mediated by inhibition of carbonic anhydrase in the proximal tubule, resulting in reduced proximal sodium uptake and consequently-via tubuloglomerular feedback一to decreased glomerular filtration. They also found that in mpkCCD cells treated with lithium, hydrochlorothiazide administration led to an increase in Aqp2 abundance at the apical membrane. ${ }^{101}$ However, the finding that lithium-treated and control SLC12A3 knock-out mice had similar urine osmolality after hydrochlorothiazide administration suggests that this effect is negligible in vivo.

The hypothesis of impaired proximal sodium reabsorption as a mechanism for reduced urine volume in $\mathrm{NDI}^{101}$ is surprising and in direct contrast to the earlier hypothesis that enhanced proximal sodium uptake leads to enhanced proximal water reabsorption. ${ }^{100}$ To what degree these apparently opposing mechanisms occur in patients remains to be determined.

\section{[H2] Prostaglandin synthesis inhibitors}

An effect of prostaglandins on epithelial water permeability was noted soon after their discovery. ${ }^{102}$ With the advent of inhibitors of prostaglandin synthesis, such as indomethacin, experiments showed a synergistic effect of these new drugs with AVP.103 An early study in an animal model of diabetes insipidus demonstrated that indomethacin reduced water diuresis independent of AVP.104 A similar effect of prostaglandin synthesis inhibitors was noted in patients with NDI. ${ }^{105-108}$ 
Prostaglandin synthesis inhibitors have become essential components in the treatment of NDI, particularly in the first years of life when management is the most complicated. The effect of these drugs can be quite marked when first initiated. Indeed, hyponatraemic seizures associated with rapid lowering of plasma sodium levels as a result of commencement of indomethacin and hydrochlorothiazide have been reported. ${ }^{109}$ In our experience, urine osmolality is unchanged after indomethacin treatment, suggesting that the drug acts mainly by enhancing the proximal reabsorption of salt and water. ${ }^{110}$

\section{[H1] Future outlook}

\section{[H2] Gene discovery}

Since the discovery of the two known NDI disease genes, $A V P R 2$ and $A Q P 2$, in the 1990s (Figure 4) no additional causative genes have been reported. Mutations in AVPR2 or AQP2 are not, however, found in all patients. Between 2003 and 2013, the Montreal laboratory performed genetic testing for NDI in 208 families and identified causative mutations in AQP2 in 18 families and in AVPR2 in 119 families (D. Bichet unpublished data). In some of the samples, mutations in genes causing renal disorders with secondary forms of NDI, such as Bartter syndrome were found, whereas in others, the phenotype was not detailed enough to clearly establish a diagnosis of NDI. ${ }^{56}$ In a Japanese study no mutations were identified in 7 of $62(11 \%)$ families with clinical NDI, ${ }^{34}$ suggesting that genes other than AQP2 and AVPR2 might be involved. However the phenotypes of these patients were poorly described and not further investigated by the referring physicians. 
In our clinical experience very few patients with a convincing NDI phenotype have no identified mutation. In our Great Ormond Street cohort, we have identified three such patients from two families (unpublished data). The results of our linkage analyses in these families were, however, consistent with the presence of mutations in $A V P R 2$ or $A Q P 2$. Thus, if additional NDI disease genes are identified, they are likely to be causative only in a small subset of patients.

\section{[H2] Novel treatments}

The promise of molecular medicine is that through improved understanding of specific molecular defects in individual patients, we can develop targeted, rational treatments. ${ }^{111}$ Unfortunately, progress has been slow at best. Although several novel treatment approaches for patients with congenital NDI have been suggested, little clinical data are available. These novel approaches focus on restoring AVP signalling upstream of AQP2 so are not applicable to patients with $A Q P 2$ mutations.

\section{[H3] AVPR2 antagonists}

A new treatment strategy for NDI focuses on the fact that most patients carry missense mutations in AVPR2 that result in misfolding of AVPR2 and its retention in the ER. ${ }^{47}$ If proper folding is induced using a molecular chaperone, the mutant receptor can escape the ER, traffic to the cell membrane and provide normal AVP signalling.

Cell-permeable AVPR2 antagonists are molecular chaperones that fit into the AVP binding pocket of AVPR2 and induce proper folding of the receptor. In patients with suitable mutations, these antagonists might provide a new treatment approach for NDI. The problem with this strategy is that the tighter 
the AVPR2 antagonists fit into the binding pocket, the better they promote surface expression of the receptor, but the less likely they are to diffuse off the receptor and enable normal AVP signalling. Conversely, compounds with low affinity for AVPR2 are less efficient at promoting surface expression, but are more likely to diffuse off the receptors that traffic to the cell surface. Antagonists with intermediate affinity have the best overall efficacy, but one such agent, the orally active, nonpeptide AVPR1A antagonist SR49059, only moderately reduced urine volume in five patients with X-linked NDI. ${ }^{112}$ SR49059 is no longer provided by the pharmaceutical company because of the possibility of idiosyncratic increases in liver enzymes (these increases were not observed in patients with NDI who received the drug) and no further clinical data on this treatment approach have been published.

\section{[H3] AVPR2 agonists}

Cell-permeable agonists that stimulate AVPR2 independent of AVP are another potential treatment approach for patients with NDI. As these agents do not block AVPR2 signalling they could potentially be a more effective treatment approach than AVPR2 antagonists.

\section{[H3] Vasopressin analogues}

A mutation-specific treatment strategy has also been suggested for patients with the Asn321Lys mutation in AVPR2.113 The mutant receptor seems to have normal cell surface expression in vitro, but severely decreased affinity for vasopressin and DDAVP. AVP signalling via this receptor could reportedly be rescued using the vasopressin analogue $\mathrm{Val}^{4}$-desmopressin. Clinical data on the use of this compound in patients with NDI bearing this mutation have not yet been reported. 


\section{[H3] Prostaglandin receptor agonists}

Another novel treatment approach for patients with AVPR2 mutations focuses on enhancing cAMP production in the principal cells independent of AVPR2, for example via stimulation of prostaglandin E2 receptors coupled to adenylyl cyclase (Figure 3). Such a strategy would not benefit patients with the autosomal form of NDI, as $A Q P 2$ mutations result in a defect downstream of cAMP production.

In a mouse model of X-linked diabetes insipidus with conditional deletion of the Avpr2 gene, administration of a prostaglandin E2 receptor EP4 subtype (EP4 receptor) agonist resulted in an increase in urine concentration. ${ }^{114}$ The peak increase in urine concentration (from $150 \mathrm{mOsm} / \mathrm{kg}$ to a maximum of $500 \mathrm{mOsm} / \mathrm{kg}$ ) was short lived (3 h), possibly due to down regulation of EP4 receptors in response to the high osmolality, but with mini-pump constant infusions a sustained increase in urine osmolality from $200 \mathrm{~m} 0 \mathrm{smol} / \mathrm{kg}$ to $300 \mathrm{mOsmol} / \mathrm{kg}$ was observed.

Doubling of urine osmolality in patients with NDI would halve urine output (to $\sim 61$ daily in adults) so would constitute successful treatment. In a rat model of NDI secondary to the administration of the AVPR2 antagonist, butaprost, an EP2 receptor agonist approximately doubled urine osmolality. ${ }^{115}$ To date, no clinical data on the use of prostaglandin receptor agonists in patients with NDI have been reported. Moreover, given the proven efficacy of prostaglandin synthesis inhibitors in NDI treatment, doubts remain as to whether this approach would be a viable treatment option. ${ }^{116}$ 


\section{[H3] Secretin receptor agonists}

The secretin receptor is a G-protein coupled receptor expressed in principal cells. Stimulation of this receptor can increase cAMP levels. ${ }^{117}$ Infusion of secretin and fluvastatin resulted in doubling in urine osmolality in mice with conditional deletion of the Avpr2 gene. ${ }^{118}$ If appropriate scientific and ethical permission was obtained, short-term therapy with a combination of secretin and fluvastatin could potentially be tested in humans with X-linked NDI. Long-term secretin treatment might be limited by adverse effects of the drug.

\section{[H3] cGMP phosphodiesterase inhibitors}

Increased levels of cyclic guanosine monophosphate (cGMP) have been shown to enhance trafficking of AQP2 to the luminal membrane and induce a short-lived increase in urine osmolality in Brattleboro rats (a model of cerebral diabetes insipidus with deficient vasopressin production by the hypothalamus). ${ }^{119}$ This finding suggests a potential role of cGMP phosphodiesterase inhibitors, such as sildenafil, in the treatment of patients with NDI resulting from AVPR2 mutations.

\section{[H3] Gene therapy}

Genome editing of somatic tissue or embryos is of considerable recent interest to correct defective genes in humans but the ethics, especially of embryo editing is controversial 120 121.. Currently, no such endeavours have reached the clinical arena. We anticipate that the ethical governance, safety and long term effects of such editing therapies will be determined possibly within the next two decades so that patients with hereditary disorders, such as NDI will benefit from them.

\section{[H1] Conclusions}

Our understanding of the molecular physiology of urine concentration has advanced tremendously in the past 25 years. The study of patients with inherited 
NDI has identified AVPR2 and AQP2 as key proteins required for the regulation of water permeability in the collecting duct. Other tubular disorders, such as Bartter syndrome, can present with a secondary form of NDI, which might cause diagnostic confusion. In adult nephrology practice, the majority of patients with NDI have an acquired form of the disease owing to lithium treatment. Current treatment of congenital NDI focuses on dietary modification, thiazides and prostaglandin synthesis inhibitors. Novel therapies, such as mutation-specific treatment using molecular chaperones, have been investigated in animal models, but few data from clinical studies are currently available. The aim of these treatment approaches is amelioration of the clinical phenotype of NDI. In the future, gene therapy approaches involving kidney-specific delivery of wild-type $A V P R 2$ or $A Q P 2$ could potentially enable long-term cure of congenital NDI.

1. Stevens, L.A., Coresh, J., Greene, T. \& Levey, A.S. Assessing kidney function-measured and estimated glomerular filtration rate. $N$ Engl J Med 354, 2473-83 (2006).

2. Nielsen, S., Marples, D., Frokiaer, J., Knepper, M. \& Agre, P. The aquaporin family of water channels in kidney: an update on physiology and pathophysiology of aquaporin-2. Kidney Int 49, 1718-23 (1996).

3. Nielsen, S. et al. Aquaporin-1 water channels in short and long loop descending thin limbs and in descending vasa recta in rat kidney. Am J Physiol 268, F1023-37 (1995).

4. Dantzler, W.H., Layton, A.T., Layton, H.E. \& Pannabecker, T.L. Urineconcentrating mechanism in the inner medulla: function of the thin limbs of the loops of Henle. Clin J Am Soc Nephrol 9, 1781-9 (2014).

5. Halperin, M.L., Kamel, K.S. \& Oh, M.S. Mechanisms to concentrate the urine: an opinion. Curr Opin Nephrol Hypertens 17, 416-22 (2008).

6. Pannabecker, T.L. Structure and function of the thin limbs of the loop of Henle. Compr Physiol 2, 2063-86 (2012).

7. Obermuller, N., Kunchaparty, S., Ellison, D.H. \& Bachmann, S. Expression of the $\mathrm{Na}-\mathrm{K}-2 \mathrm{Cl}$ cotransporter by macula densa and thick ascending limb cells of rat and rabbit nephron. J Clin Invest 98, 635-40 (1996).

8. Bichet, D.G., Oksche, A. \& Rosenthal, W. Congenital nephrogenic diabetes insipidus. J Am Soc Nephrol 8, 1951-8 (1997).

9. Nielsen, S. et al. Aquaporins in the kidney: from molecules to medicine. Physiol Rev 82, 205-44 (2002).

10. Kortenoeven, M.L. \& Fenton, R.A. Renal aquaporins and water balance disorders. Biochim Biophys Acta 1840, 1533-49 (2014). 
11. Fushimi, K., Sasaki, S. \& Marumo, F. Phosphorylation of serine 256 is required for cAMP-dependent regulatory exocytosis of the aquaporin-2 water channel. J Biol Chem 272, 14800-4 (1997).

12. Kamsteeg, E.J., Heijnen, I., van Os, C.H. \& Deen, P.M. The subcellular localization of an aquaporin-2 tetramer depends on the stoichiometry of phosphorylated and nonphosphorylated monomers. J Cell Biol 151, 91930 (2000).

13. Mulders, S.M. et al. An aquaporin-2 water channel mutant which causes autosomal dominant nephrogenic diabetes insipidus is retained in the Golgi complex. J Clin Invest 102, 57-66 (1998).

14. Savelkoul, P.J. et al. p.R254Q mutation in the aquaporin-2 water channel causing dominant nephrogenic diabetes insipidus is due to a lack of arginine vasopressin-induced phosphorylation. Hum Mutat 30, E891-903 (2009).

15. Hoffert, J.D. et al. Vasopressin-stimulated increase in phosphorylation at Ser269 potentiates plasma membrane retention of aquaporin-2.J Biol Chem 283, 24617-27 (2008).

16. Moeller, H.B., Praetorius, J., Rutzler, M.R. \& Fenton, R.A. Phosphorylation of aquaporin-2 regulates its endocytosis and protein-protein interactions. Proc Natl Acad Sci U S A 107, 424-9 (2010).

17. Tamma, G., Robben, J.H., Trimpert, C., Boone, M. \& Deen, P.M. Regulation of AQP2 localization by S256 and S261 phosphorylation and ubiquitination. Am J Physiol Cell Physiol 300, C636-46 (2011).

18. Kamsteeg, E.J. et al. Short-chain ubiquitination mediates the regulated endocytosis of the aquaporin-2 water channel. Proc Natl Acad Sci U S A 103, 18344-9 (2006).

19. Rehmann, H., Wittinghofer, A. \& Bos, J.L. Capturing cyclic nucleotides in action: snapshots from crystallographic studies. Nat Rev Mol Cell Biol 8, 63-73 (2007).

20. Holz, G.G., Kang, G., Harbeck, M., Roe, M.W. \& Chepurny, O.G. Cell physiology of cAMP sensor Epac. J Physiol 577, 5-15 (2006).

21. Kortenoeven, M.L. et al. In mpkCCD cells, long-term regulation of aquaporin-2 by vasopressin occurs independent of protein kinase $\mathrm{A}$ and CREB but may involve Epac. Am J Physiol Renal Physiol 302, F1395-401 (2012).

22. Hozawa, S., Holtzman, E.J. \& Ausiello, D.A. cAMP motifs regulating transcription in the aquaporin 2 gene. Am J Physiol 270, C1695-702 (1996).

23. Matsumura, Y., Uchida, S., Rai, T., Sasaki, S. \& Marumo, F. Transcriptional regulation of aquaporin-2 water channel gene by cAMP. J Am Soc Nephrol 8, 861-7 (1997).

24. Yasui, M., Zelenin, S.M., Celsi, G. \& Aperia, A. Adenylate cyclase-coupled vasopressin receptor activates AQP2 promoter via a dual effect on CRE and AP1 elements. Am J Physiol 272, F443-50 (1997).

25. Hasler, U. et al. Long term regulation of aquaporin-2 expression in vasopressin-responsive renal collecting duct principal cells. J Biol Chem 277, 10379-86 (2002). 
26. Terris, J., Ecelbarger, C.A., Nielsen, S. \& Knepper, M.A. Long-term regulation of four renal aquaporins in rats. Am J Physiol 271, F414-22 (1996).

27. Lolait, S.J. et al. Cloning and characterization of a vasopressin V2 receptor and possible link to nephrogenic diabetes insipidus. Nature 357, 336-9 (1992).

28. Rosenthal, W. et al. Molecular identification of the gene responsible for congenital nephrogenic diabetes insipidus. Nature 359, 233-5 (1992).

29. van den Ouweland, A.M. et al. Mutations in the vasopressin type 2 receptor gene (AVPR2) associated with nephrogenic diabetes insipidus. Nat Genet 2, 99-102 (1992).

30. Pan, Y., Metzenberg, A., Das, S., Jing, B. \& Gitschier, J. Mutations in the V2 vasopressin receptor gene are associated with $\mathrm{X}$-linked nephrogenic diabetes insipidus. Nat Genet 2, 103-6 (1992).

31. Fushimi, K. et al. Cloning and expression of apical membrane water channel of rat kidney collecting tubule. Nature 361, 549-52 (1993).

32. Sasaki, S. et al. Cloning, characterization, and chromosomal mapping of human aquaporin of collecting duct. J Clin Invest 93, 1250-6 (1994).

33. Deen, P.M. et al. Requirement of human renal water channel aquaporin-2 for vasopressin-dependent concentration of urine. Science 264, 92-5 (1994).

34. Sasaki, S., Chiga, M., Kikuchi, E., Rai, T. \& Uchida, S. Hereditary nephrogenic diabetes insipidus in Japanese patients: analysis of 78 families and report of 22 new mutations in AVPR2 and AQP2. Clin Exp Nephrol 17, 338-44 (2013).

35. Arthus, M.F. et al. Report of 33 novel AVPR2 mutations and analysis of 117 families with X-linked nephrogenic diabetes insipidus. J Am Soc Nephrol 11, 1044-54 (2000).

36. Sands, J.M. \& Bichet, D.G. Nephrogenic diabetes insipidus. Ann Intern Med 144, 186-94 (2006).

37. Wesche, D., Deen, P.M. \& Knoers, N.V. Congenital nephrogenic diabetes insipidus: the current state of affairs. Pediatr Nephrol 27, 2183-204 (2012).

38. Bichet, D.G. Nephrogenic diabetes insipidus. Adv Chronic Kidney Dis 13, 96-104 (2006).

39. van Lieburg, A.F., Knoers, N.V. \& Monnens, L.A. Clinical presentation and follow-up of 30 patients with congenital nephrogenic diabetes insipidus. $J$ Am Soc Nephrol 10, 1958-64 (1999).

40. Sato, K. et al. A novel mutation in the vasopressin V2 receptor gene in a woman with congenital nephrogenic diabetes insipidus. Intern Med 38, 808-12 (1999).

41. Kinoshita, K. et al. A novel deletion mutation in the arginine vasopressin receptor 2 gene and skewed $X$ chromosome inactivation in a female patient with congenital nephrogenic diabetes insipidus. J Endocrinol Invest 27, 167-70 (2004).

42. Satoh, M., Ogikubo, S. \& Yoshizawa-Ogasawara, A. Correlation between clinical phenotypes and X-inactivation patterns in six female carriers with heterozygote vasopressin type 2 receptor gene mutations. Endocr J 55, 277-84 (2008). 
43. Bockenhauer, D. et al. Vasopressin type 2 receptor V88M mutation: molecular basis of partial and complete nephrogenic diabetes insipidus. Nephron Physiol 114, p1-10 (2010).

44. Bichet, D.G. et al. X-linked nephrogenic diabetes insipidus mutations in North America and the Hopewell hypothesis. J Clin Invest 92, 1262-8 (1993).

45. Fujiwara, T.M. \& Bichet, D.G. Molecular biology of hereditary diabetes insipidus. J Am Soc Nephrol 16, 2836-46 (2005).

46. Spanakis, E., Milord, E. \& Gragnoli, C. AVPR2 variants and mutations in nephrogenic diabetes insipidus: review and missense mutation significance. J Cell Physiol 217, 605-17 (2008).

47. Morello, J.P. et al. Pharmacological chaperones rescue cell-surface expression and function of misfolded V2 vasopressin receptor mutants. $J$ Clin Invest 105, 887-95 (2000).

48. Duzenli, D. et al. Mutations in the AVPR2, AVP-NPII, and AQP2 genes in Turkish patients with diabetes insipidus. Endocrine 42, 664-9 (2012).

49. Marr, N. et al. Heteroligomerization of an Aquaporin-2 mutant with wildtype Aquaporin-2 and their misrouting to late endosomes/lysosomes explains dominant nephrogenic diabetes insipidus. Hum Mol Genet 11, 779-89 (2002).

50. Kuwahara, M. et al. Three families with autosomal dominant nephrogenic diabetes insipidus caused by aquaporin-2 mutations in the $\mathrm{C}$-terminus. Am J Hum Genet 69, 738-48 (2001).

51. Kamsteeg, E.J. et al. Reversed polarized delivery of an aquaporin-2 mutant causes dominant nephrogenic diabetes insipidus. J Cell Biol 163, 1099109 (2003).

52. Frick, A. et al. X-ray structure of human aquaporin 2 and its implications for nephrogenic diabetes insipidus and trafficking. Proc Natl Acad Sci U S A 111, 6305-10 (2014).

53. Bichet, D.G. et al. Aquaporin-2: new mutations responsible for autosomalrecessive nephrogenic diabetes insipidus - update and epidemiology. Clin Kidney J 5, 195-202 (2012).

54. Bichet, D.G. et al. Aquaporin-2: new mutations responsible for autosomalrecessive nephrogenic diabetes insipidus - update and epidemiology. Clinical Kidney Journal 5, 195-202 (2012).

55. Bockenhauer, D. et al. Secondary nephrogenic diabetes insipidus as a complication of inherited renal diseases. Nephron Physiol 116, p23-9 (2010).

56. Bockenhauer, D. \& Bichet, D.G. Inherited secondary nephrogenic diabetes insipidus: concentrating on humans. Am J Physiol Renal Physiol 304, F1037-42 (2013).

57. Bettinelli, A. et al. Phenotypic variability in Bartter syndrome type I. Pediatr Nephrol 14, 940-5 (2000).

58. Hebert, S.C., Brown, E.M. \& Harris, H.W. Role of the $\mathrm{Ca}(2+)$-sensing receptor in divalent mineral ion homeostasis. J Exp Biol 200, 295-302 (1997).

59. Marples, D., Frokiaer, J., Dorup, J., Knepper, M.A. \& Nielsen, S. Hypokalemia-induced downregulation of aquaporin-2 water channel 
expression in rat kidney medulla and cortex. J Clin Invest 97, 1960-8 (1996).

60. Sands, J.M. et al. Apical extracellular calcium/polyvalent cation-sensing receptor regulates vasopressin-elicited water permeability in rat kidney inner medullary collecting duct. J Clin Invest 99, 1399-405 (1997).

61. Earm, J.H. et al. Decreased aquaporin-2 expression and apical plasma membrane delivery in kidney collecting ducts of polyuric hypercalcemic rats. J Am Soc Nephrol 9, 2181-93 (1998).

62. Trepiccione, F. \& Christensen, B.M. Lithium-induced nephrogenic diabetes insipidus: new clinical and experimental findings. J Nephrol 23 Suppl 16, S43-8 (2010).

63. Rej, S., Herrmann, N. \& Shulman, K. The effects of lithium on renal function in older adults--a systematic review. Journal of geriatric psychiatry and neurology 25, 51-61 (2012).

64. Timmer, R.T. \& Sands, J.M. Lithium intoxication. J Am Soc Nephrol 10, 66674 (1999).

65. Hetmar, O. et al. Lithium: long-term effects on the kidney. I. Renal function in retrospect. Acta Psychiatr Scand 73, 574-81 (1986).

66. Juurlink, D.N. et al. Drug-induced lithium toxicity in the elderly: a population-based study. J Am Geriatr Soc 52, 794-8 (2004).

67. Head, L. \& Dening, T. Lithium in the over-65s: who is taking it and who is monitoring it? A survey of older adults on lithium in the Cambridge Mental Health Services catchment area. Int J Geriatr Psychiatry 13, 164-71 (1998).

68. Christensen, B.M. et al. Changes in cellular composition of kidney collecting duct cells in rats with lithium-induced NDI. Am J Physiol Cell Physiol 286, C952-64 (2004).

69. Li, Y., Shaw, S., Kamsteeg, E.J., Vandewalle, A. \& Deen, P.M. Development of lithium-induced nephrogenic diabetes insipidus is dissociated from adenylyl cyclase activity. J Am Soc Nephrol 17, 1063-72 (2006).

70. Walker, R.J. et al. Lithium-induced reduction in urinary concentrating ability and urinary aquaporin 2 (AQP2) excretion in healthy volunteers. Kidney Int 67, 291-4 (2005).

71. Kortenoeven, M.L. et al. Amiloride blocks lithium entry through the sodium channel thereby attenuating the resultant nephrogenic diabetes insipidus. Kidney Int 76, 44-53 (2009).

72. Bedford, J.J. et al. Lithium-induced nephrogenic diabetes insipidus: renal effects of amiloride. Clin J Am Soc Nephrol 3, 1324-31 (2008).

73. Batlle, D.C., von Riotte, A.B., Gaviria, M. \& Grupp, M. Amelioration of polyuria by amiloride in patients receiving long-term lithium therapy. $N$ Engl J Med 312, 408-14 (1985).

74. Frokiaer, J., Marples, D., Knepper, M.A. \& Nielsen, S. Bilateral ureteral obstruction downregulates expression of vasopressin-sensitive AQP-2 water channel in rat kidney. Am J Physiol 270, F657-68 (1996).

75. Frokiaer, J. et al. Downregulation of aquaporin-2 parallels changes in renal water excretion in unilateral ureteral obstruction. Am J Physiol 273, F213-23 (1997).

76. Winberg, J. Determination of Renal Concentration Capacity in Infants and Children without Renal Disease. Acta Paediatrica 48, 318-328 (1958). 
77. Bichet, D.G. et al. Hemodynamic and coagulation responses to 1desamino[8-D-arginine] vasopressin in patients with congenital nephrogenic diabetes insipidus. $N$ Engl J Med 318, 881-7 (1988).

78. Mannucci, P.M. Treatment of von Willebrand's Disease. N Engl J Med 351, 683-94 (2004).

79. Kaufmann, J.E. \& Vischer, U.M. Cellular mechanisms of the hemostatic effects of desmopressin (DDAVP). J Thromb Haemost 1, 682-9 (2003).

80. Hillman, D.A., Neyzi, O., Porter, P., Cushman, A. \& Talbot, N.B. Renal (vasopressin-resistant) diabetes insipidus; definition of the effects of a homeostatic limitation in capacity to conserve water on the physical, intellectual and emotional development of a child. Pediatrics 21, 430-5 (1958).

81. Vest, M., Talbotnb \& Crawford, J.D. Hypocaloric dwarfism and hydronephrosis in diabetes insipidus. Am J Dis Child 105, 175-81 (1963).

82. Schofer, 0. et al. Nephrogenic diabetes insipidus and intracerebral calcification. Arch Dis Child 65, 885-7 (1990).

83. Hoekstra, J.A., van Lieburg, A.F., Monnens, L.A., Hulstijn-Dirkmaat, G.M. \& Knoers, V.V. Cognitive and psychosocial functioning of patients with congenital nephrogenic diabetes insipidus. Am J Med Genet 61, 81-8 (1996).

84. Huber, D., Veinante, P. \& Stoop, R. Vasopressin and oxytocin excite distinct neuronal populations in the central amygdala. Science 308, 245-8 (2005).

85. Griebel, G. et al. Anxiolytic- and antidepressant-like effects of the nonpeptide vasopressin V1b receptor antagonist, SSR149415, suggest an innovative approach for the treatment of stress-related disorders. Proc Natl Acad Sci U S A 99, 6370-5 (2002).

86. Yoo, T.H. et al. Congenital Nephrogenic Diabetes Insipidus Presented with Bilateral Hydronephrosis: Genetic Analysis of V2R Gene Mutations. Yonsei Med J 47, 126-30 (2006).

87. Stevens, S., Brown, B.D. \& McGahan, J.P. Nephrogenic diabetes insipidus: a cause of severe nonobstructive urinary tract dilatation. J Ultrasound Med 14, 543-5 (1995).

88. Jaureguiberry, G. et al. A patient with polyuria and hydronephrosis: question. Pediatric Nephrology 26, 1977-8, 1979-80 (2011).

89. Sadeghi, H., Robertson, G.L., Bichet, D.G., Innamorati, G. \& Birnbaumer, M. Biochemical basis of partial nephrogenic diabetes insipidus phenotypes. Mol Endocrinol 11, 1806-13 (1997).

90. Canfield, M.C., Tamarappoo, B.K., Moses, A.M., Verkman, A.S. \& Holtzman, E.J. Identification and characterization of aquaporin-2 water channel mutations causing nephrogenic diabetes insipidus with partial vasopressin response. Hum Mol Genet 6, 1865-71 (1997).

91. Guyon, C. et al. Characterization of D150E and G196D aquaporin-2 mutations responsible for nephrogenic diabetes insipidus: importance of a mild phenotype. Am J Physiol Renal Physiol 297, F489-98 (2009).

92. Bichet, D.G. et al. Nature and recurrence of AVPR2 mutations in X-linked nephrogenic diabetes insipidus. Am J Hum Genet 55, 278-86 (1994).

93. Ala, Y. et al. Functional studies of twelve mutant V2 vasopressin receptors related to nephrogenic diabetes insipidus: molecular basis of a mild clinical phenotype. J Am Soc Nephrol 9, 1861-72 (1998). 
94. Kahn, A., Brachet, E. \& Blum, D. Controlled fall in natremia and risk of seizures in hypertonic dehydration. Intensive Care Med 5, 27-31 (1979).

95. Cansick, J., Rees, L., Koffman, G., Van't Hoff, W. \& Bockenhauer, D. A fatal case of cerebral oedema with hyponatraemia and massive polyuria after renal transplantation. Pediatric Nephrology 24, 1231-4 (2009).

96. Sterns, R.H. Disorders of plasma sodium - causes, consequences, and correction. $N$ Engl J Med 372, 55-65 (2015).

97. Fang, C. et al. Fluid management of hypernatraemic dehydration to prevent cerebral oedema: a retrospective case control study of 97 children in China. J Paediatr Child Health 46, 301-3 (2010).

98. Meyer, E. Über Diabetes insipidus und andere Polyurien. Deutsch. Arch. klin. Med. 83, 1 (1905).

99. Crawford, J.D. \& Kennedy, G.C. Chlorothiazid in diabetes insipidus. Nature 183, 891-2 (1959).

100. Havard, C.W. Thiazide-induced antidiuresis in diabetes insipidus. Proc $R$ Soc Med 58, 1005-7 (1965).

101. Sinke, A.P. et al. Hydrochlorothiazide attenuates lithium-induced nephrogenic diabetes insipidus independently of the sodium-chloride cotransporter. Am J Physiol Renal Physiol 306, F525-33 (2014).

102. Orloff, J., Handler, J.S. \& Bergstrom, S. Effect of Prostaglandin (Pge-1) on the Permeability Response of Toad Bladder to Vasopressin, Theophylline and Adenosine 3',5'-Monophosphate. Nature 205, 397-8 (1965).

103. Anderson, R.J., Berl, T., McDonald, K.D. \& Schrier, R.W. Evidence for an in vivo antagonism between vasopressin and prostaglandin in the mammalian kidney. J Clin Invest 56, 420-6 (1975).

104. Stoff, J.S., Rosa, R.M., Silva, P. \& Epstein, F.H. Indomethacin impairs water diuresis in the DI rat: role of prostaglandins independent of ADH. Am J Physiol 241, F231-7 (1981).

105. Libber, S., Harrison, H. \& Spector, D. Treatment of nephrogenic diabetes insipidus with prostaglandin synthesis inhibitors. J Pediatr 108, 305-11 (1986).

106. Monnens, L., Jonkman, A. \& Thomas, C. Response to indomethacin and hydrochlorothiazide in nephrogenic diabetes insipidus. Clin Sci (Lond) 66, 709-15 (1984).

107. Monn, E. Prostaglandin synthetase inhibitors in the treatment of nephrogenic diabetes insipidus. Acta Paediatr Scand 70, 39-42 (1981).

108. Usberti, M. et al. Renal prostaglandin E2 in nephrogenic diabetes insipidus: effects of inhibition of prostaglandin synthesis by indomethacin. J Pediatr 97, 476-8 (1980).

109. Boussemart, T., Nsota, J., Martin-Coignard, D. \& Champion, G. Nephrogenic diabetes insipidus: treat with caution. Pediatr Nephrol 24, 1761-3 (2009).

110. Bockenhauer, D. et al. Antenatal Bartter's syndrome: why is this not a lethal condition? QJM : monthly journal of the Association of Physicians 101, 927-42 (2008).

111. Bockenhauer, D., Medlar, A.J., Ashton, E., Kleta, R. \& Lench, N. Genetic testing in renal disease. Pediatric Nephrology 27, 873-83 (2012).

112. Bernier, V. et al. Pharmacologic chaperones as a potential treatment for Xlinked nephrogenic diabetes insipidus. J Am Soc Nephrol 17, 232-43 (2006). 
113. Erdelyi, L.S. et al. Altered agonist sensitivity of a mutant v2 receptor suggests a novel therapeutic strategy for nephrogenic diabetes insipidus. Mol Endocrinol 28, 634-43 (2014).

114. Li, J.H. et al. A selective EP4 PGE2 receptor agonist alleviates disease in a new mouse model of X-linked nephrogenic diabetes insipidus. J Clin Invest 119, 3115-26 (2009).

115. Olesen, E.T., Rutzler, M.R., Moeller, H.B., Praetorius, H.A. \& Fenton, R.A. Vasopressin-independent targeting of aquaporin-2 by selective $\mathrm{E}$ prostanoid receptor agonists alleviates nephrogenic diabetes insipidus. Proc Natl Acad Sci U S A 108, 12949-54 (2011).

116. Bockenhauer, D. \& Bichet, D.G. Urinary concentration: different ways to open and close the tap. Pediatr Nephrol 29, 1297-303 (2014).

117. Chu, J.Y. et al. Phenotypes developed in secretin receptor-null mice indicated a role for secretin in regulating renal water reabsorption. $\mathrm{Mol}$ Cell Biol 27, 2499-511 (2007).

118. Procino, G. et al. Combination of secretin and fluvastatin ameliorates the polyuria associated with X-linked nephrogenic diabetes insipidus in mice. Kidney Int 86, 127-38 (2014).

119. Bouley, R. et al. Stimulation of AQP2 membrane insertion in renal epithelial cells in vitro and in vivo by the cGMP phosphodiesterase inhibitor sildenafil citrate (Viagra). Am J Physiol Renal Physiol 288, F110312 (2005).

120. Ran, F.A. et al. In vivo genome editing using Staphylococcus aureus Cas9. Nature 520, 186-91 (2015).

121. Cyranoski, D. Ethics of embryo editing divides scientists. Nature 519, 272 (2015).

122. Coleman, J. in Clinical Paediatric Dietetics (eds. Shaw, V. \& Lawson, M.) (Blackwell Science Ltd, Oxford, 2001). 


\section{Box 1: Osmotic load}

The osmotic load consists of osmotically active substances that need to be excreted in the urine. The key contributors to the osmotic load are proteins, which are metabolized to urea (approximately $4 \mathrm{mmol}$ per g of protein) and salts. A typical western diet contains about 800 m0sm per day. An individual with a urine osmolality of $800 \mathrm{~m} 0 \mathrm{sm} / \mathrm{kg}$ only needs $1 \mathrm{l}$ of water to excrete this osmotic load, whereas a patient with maximal urine osmolality of $100 \mathrm{~m} 0 \mathrm{sm} / \mathrm{kg}$ will need at least $8 \mathrm{l}$ of water for excretion. $1 \mathrm{~g}$ of table salt providing an osmolar load of $\sim 36$ mOsm (18 mOsm Na and 18 mOsm Cl). Consequently, in a patient with a urine osmolality of $100 \mathrm{mOsm} / \mathrm{kg}$, each gram of salt ingested will increase the obligatory urine output by $360 \mathrm{ml}$. The osmolar load of a diet can be roughly estimated by multiplying the millimolar amounts of sodium and potassium by two (to account for the accompanying anions) and adding the amount of protein in g multiplied by four. ${ }^{122}$ As metabolism of lipids and sugars does not produce byproducts that require renal excretion, they do not contribute to the osmotic load. Diet is the key contributor to the osmotic load, but metabolism of own proteins during catabolic states also generate urea.

Figure 1: The renal concentration and dilution mechanism. The loop of Henle forms a counter-current multiplier system that concentrates the urine. Urine is isotonic when it enters the loop of Henle and hypotonic when it exits into the collecting duct. The concentration gradient generated in the loop of Henle is driven by the active reabsorption of $\mathrm{NaCl}$ in the thick ascending limb by the transporter solute carrier family 12 member 1 . The mechanism of concentration in the thin descending limb is not completely resolved, but likely 
involves passive water efflux and/or $\mathrm{NaCl}$ influx. Final concentration of urine occurs in the collecting duct and depends on the availability of aquaporin 2 water channels. The approximate osmolalities of the tubular fluid (pink boxes) and interstitial fluid (green boxes) are indicated.

Figure 2: Regulation of vasopressin secretion. a | The concentration of various ions in the blood is continuously monitored by sensory neurons located in brain regions such as the subfornical organ, median preoptic nucleus and organum vasculosum lamina terminus. ${ }^{123}$ These sensory neurons track osmotic conditions and signal any perturbations to magnocellular neurosecretory cells in the supraoptic nuclei and paraventricular nuclei in the hypothalamus. The magnocellular neurosecretory cells then go on to produce and release vasopressin into the circulation through the posterior pituitary. The autonomic nervous system also regulates kidney function. b | Cell autonomous osmoreception in vasopressin neurons. Changes in osmolality cause inversely proportional changes in soma volume. Shrinkage activates TRPV1 channels and the ensuing depolarization increases action potential firing rate and vasopressin release from axon terminals in the neurohypophysis. Increased vasopressin levels in blood enhance water reabsorption by the kidney (antidiuresis) to restore extracellular fluid osmolality toward the set point. Hypotonic stimuli inhibit TRPV1. The resulting hyperpolarization and inhibition of firing reduces vasopressin release and promotes diuresis. Abbreviation: TRPV1, transient receptor vanilloid-type 1. Part a modified with permission from Nature Publishing Group (C) Colwell, C. S. Preventing dehydration during sleep. Nat. Neurosci. 13, 403-404 (2010). Part b modified with permission from John Wiley \& Sons Ltd (C) Prager-Khoutorsky, M. \& Bourque, C. W. Mechanical basis of osmosensory transduction in magnocellular neurosecretory neurons of the rat supraoptic nucleus. J. Neuroendocrinol. http://dx.doi.org/10.1111/jne.12270.

Figure 3: Water transport in the principal cell. Binding of AVP to the AVPR2 receptor expressed on the basolateral side of the principal cell stimulates the 


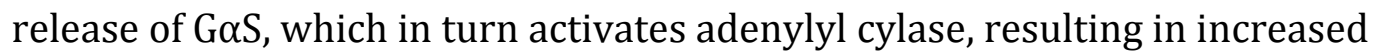
cAMP production. PKA is activated by cAMP and phosphorylates several residues in the C-terminus of AQP2. This phosphorylation enables trafficking of AQP2 to the apical membrane and the formation of AQP2 water channels. AQP2 is constantly retrieved from the membrane by endocytosis, so that ongoing water permeability depends on delivery of AQP2 to the membrane, either by recycling or generation of new channels. Water diffuses via these channels along the concentration gradient from the tubular lumen into the principal cell and exits into the interstitium via the constitutively expressed AQP3 and AQP4 water channels. In addition to PKA-mediated phosphorylation ofAQP2, cAMP enhances transcription of AQP2. Some of the changes induced by cAMP are likely mediated by EPAC. The G-protein coupled receptors EP2, EP4 and the secretin receptor are also expressed in principal cells. Targeting of these receptors could potentially be used to stimulate membrane insertion of AQP2 in the absence of functional AVPR2. Abbreviations: AQP, aquaporin; AVP, arginine vasopressin; AVPR2, vasopressin V2 receptor; CREB, cAMP responsive element binding protein; EP2, prostaglandin E2 receptor EP2 subtype; EP4, prostaglandin E2 receptor EP4 subtype; EPAC, exchange protein directly activated by cAMP; G, G protein; PKA, protein kinase A.

\section{Figure 4 | Timeline of key advances in the understanding of nephrogenic}

diabetes insipidus. Abbreviations: AQP2, aquaporin 2; AVP, arginine vasopressin; AVPR2, vasopressin V2 receptor. 